\title{
Onset of superconductivity and retention of magnetic fields in cooling neutron stars
}

\author{
Wynn C. G. Ho ${ }^{1,2}$ Nils Andersson ${ }^{1}$, and Vanessa Graber ${ }^{3}$ \\ ${ }^{1}$ Mathematical Sciences and STAG Research Centre, \\ University of Southampton, Southampton, SO17 1BJ, United Kingdom \\ email: wynnho@slac.stanford.edu \\ ${ }^{2}$ Physics and Astronomy, University of Southampton, \\ Southampton, SO17 1BJ, United Kingdom \\ ${ }^{3}$ Department of Physics and McGill Space Institute, \\ McGill University, Montreal, QC, H3A 2T8, Canada
}

\begin{abstract}
A superconductor of paired protons is thought to form in the core of neutron stars soon after their birth. Minimum energy conditions suggest that magnetic flux is expelled from the superconducting region due to the Meissner effect, such that the neutron star core retains or is largely devoid of magnetic fields for some nuclear equation of state and proton pairing models. We show via neutron star cooling simulations that the superconducting region expands faster than flux is expected to be expelled because cooling timescales are much shorter than timescales of magnetic field diffusion. Thus magnetic fields remain in the bulk of the neutron star core for at least $10^{6}-10^{7} \mathrm{yr}$. We estimate the size of flux free regions at $10^{7} \mathrm{yr}$ to be $\lesssim 100 \mathrm{~m}$ for a magnetic field of $10^{11} \mathrm{G}$ and possibly smaller for stronger field strengths.
\end{abstract}

Keywords. dense matter, magnetic fields, stars: neutron

\section{Introduction}

Neutron stars (NSs) are born hot, with temperatures well in excess of $10^{10} \mathrm{~K}$, and cool over time via neutrino and photon emission. When the local temperature $T$ falls below the proton critical temperature $T_{\mathrm{cp}}$, protons become superconducting (see, e.g., Sauls 1989, Page et al. 2014, for review). The critical temperature $T_{\mathrm{cp}}$ is related to the energy gap for Cooper singlet $\left({ }^{1} S_{0}\right)$ pairing $\Delta$ in the zero temperature limit by $k_{\mathrm{B}} T_{\mathrm{cp}} \approx 0.5669 \Delta$. Figure 1 shows the evolution of the core temperature profile from our cooling simulations (Ho et al. 2012) using the APR nuclear equation of state (Akmal et al. 1998; Page et al. 2004) and CCDK pairing gap (Chen et al. 1993) models. We see that, when a NS is only several minutes old, the temperature drops below the maximum critical temperature $\left(T<T_{\text {cp }}\right.$ at baryon density $\left.\approx 0.2 \mathrm{fm}^{-3}\right)$, such that a proton superconductor begins to form at radius $r \sim 10 \mathrm{~km}$. At subsequent times, the superconducting region grows and encompasses more of the star.

A paired proton superconductor can take two forms in the core of a NS, depending on the Ginzburg-Landau parameter $\kappa \equiv \lambda / \xi$ and two critical magnetic fields $H_{\mathrm{c} 1}=$ $\left(\phi_{0} / 4 \pi \lambda^{2}\right) \ln \kappa$ and $H_{\mathrm{c} 2}=\phi_{0} / 2 \pi \xi^{2}$, where $\phi_{0}=\pi \hbar c / e$ is the magnetic flux quantum and the equation for $H_{\mathrm{c} 1}$ is in the limit of large $\kappa$ (Tinkham 1996). The magnetic field penetration lengthscale is $\lambda=\left(m_{\mathrm{p}}^{*} c^{2} / 4 \pi e^{2} n_{\mathrm{e}}\right)^{1 / 2}$ and the superconductor pairing or coherence lengthscale (also typical size of magnetic fluxtube) is $\xi=\hbar^{2} k_{\mathrm{F}} / \pi m_{\mathrm{p}}^{*} \Delta$, where $m_{\mathrm{p}}^{*}$ is effective proton mass, $\hbar k_{\mathrm{F}}=\hbar\left(3 \pi^{2} n_{\mathrm{p}}\right)^{1 / 3}$ is Fermi momentum, and $n_{\mathrm{e}}$ and $n_{\mathrm{p}}$ are electron and proton number densities, respectively. If the Ginzburg-Landau parameter $\kappa<1 / \sqrt{2}$, then an external magnetic field $H$ does not penetrate significantly into the 

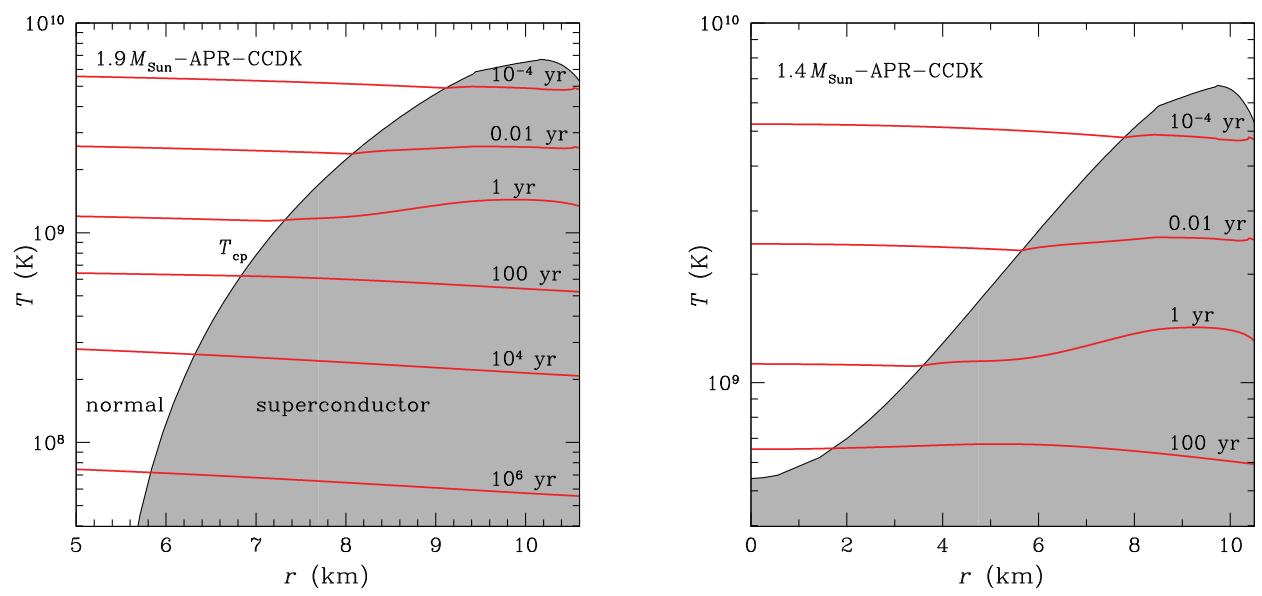

Figure 1. Temperature $T$ as a function of radius $r$. The crust-core boundary is at $r \approx 10.7 \mathrm{~km}$, and total radius is $11.2 \mathrm{~km}$ for a $1.9 M_{\mathrm{Sun}} \mathrm{NS}$ (left) and $11.6 \mathrm{~km}$ for a $1.4 M_{\mathrm{Sun}} \mathrm{NS}$ (right) built using the APR equation of state. $T_{\mathrm{cp}}$ denotes the density-dependent critical temperature for onset of proton superconductivity (when $T<T_{\mathrm{cp}}$ ) using the CCDK gap model. Nearly horizontal curves show temperature profiles at various ages.
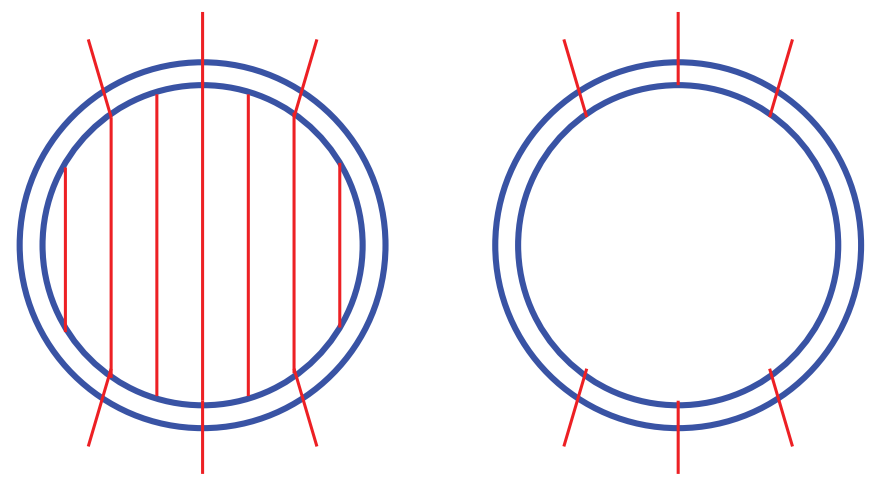

Figure 2. Schematic of magnetic field structure in neutron star core and crust. Lines represent magnetic flux which is retained in the core (left) or expelled due to the Meissner effect (right).

superconductor, and magnetic flux is expelled from superconducting regions (such that $B=0$ ) due to the Meissner effect (see, e.g., Baym et al. 1969; Sauls 1989; Tinkham 1996; Graber et al. 2017). In this state, magnetic flux can be retained in macroscopic regions of normal conducting matter that alternate with regions of flux-free superconducting matter. Conversely, if $\kappa>1 / \sqrt{2}$, then magnetic field can reside in superconducting fluxtubes. The energy of the fluxtube state is at a minimum when the magnetic field $H$ is $H_{\mathrm{c} 1} \lesssim H \lesssim H_{\mathrm{c} 2}$, with indicative values $H_{\mathrm{c} 1} \sim 10^{15} \mathrm{G}$ and $H_{\mathrm{c} 2} \sim 10^{16} \mathrm{G}$. For $H \lesssim H_{\mathrm{c} 1}$, the superconductor should be in a Meissner state (i.e., magnetic flux expulsion), while superconductivity is destroyed for $H \gtrsim H_{\mathrm{c} 2}$. In the present work, we only consider and present results for the case when $H \ll H_{\mathrm{c} 1}$, with a superconductor region that is illustrated in Fig. 1, and using $\tau_{\mathrm{OhmH}}$ (see below). Thus a large portion of the NS interior would be in the Meissner (magnetic flux-free) state once superconductivity sets in. Other cases, including for smaller superconducting regions, are examined in Ho et al. (2017). The question we address in this work and in Ho et al. (2017) is which of the two magnetic field configurations illustrated in Fig. 2 exists inside NSs. 
In order for a Meissner state to be created, magnetic field must be expelled from the superconducting region (on the flux diffusion timescale) more rapidly than the region grows (on the cooling timescale). To find the former, Baym (1970) solves the equations for flux diffusion and energy transfer at the fixed boundary between a superconducting region and a normal region. This calculation yields a (modified Ohmic) diffusion timescale (for $H / H_{\mathrm{c} 2} \ll 1$ )

$$
\tau_{\mathrm{OhmH}} \approx=\frac{4 \pi \sigma_{\mathrm{c}} l_{\mathrm{mag}}^{2}}{c^{2}} \frac{H}{2 H_{\mathrm{c} 2}}=4.4 \times 10^{6} \mathrm{yr}\left(\frac{\sigma_{\mathrm{c}}}{10^{29} \mathrm{~s}^{-1}}\right)\left(\frac{l_{\mathrm{mag}}}{1 \mathrm{~km}}\right)^{2}\left(\frac{H / 2 H_{\mathrm{c} 2}}{10^{-5}}\right),
$$

where $\tau_{\mathrm{Ohm}}$ is the magnetic field diffusion/dissipation timescale in non-superconducting matter, $\sigma_{\mathrm{c}}$ is electrical conductivity due to scattering, and $l_{\mathrm{mag}}$ is the lengthscale over which magnetic field changes.

In contrast, at ages $\lesssim 10^{6} \mathrm{yr}$, NSs cool via neutrino emission (Yakovlev \& Pethick 2004; Potekhin et al. 2015) over a timescale

$$
\tau_{\text {cool }}=C T / \epsilon_{\nu} \sim 1 \mathrm{yr}\left(n_{\mathrm{n}} / n_{\mathrm{e}}\right)^{1 / 3}\left(10^{9} \mathrm{~K} / T\right)^{6},
$$

where $C=1.6 \times 10^{20} \mathrm{erg} \mathrm{cm}^{-3} \mathrm{~K}^{-1}\left(n_{\mathrm{n}} / n_{\mathrm{nuc}}\right)^{1 / 3}\left(T / 10^{9} \mathrm{~K}\right)$ is the neutron heat capacity, $\epsilon_{\nu} \sim 3 \times 10^{22} \mathrm{erg} \mathrm{cm}^{-3} \mathrm{~s}^{-1}\left(n_{\mathrm{e}} / n_{\text {nuc }}\right)^{1 / 3}\left(T / 10^{9} \mathrm{~K}\right)^{8}$ is the neutrino emissivity for modified Urca processes, and $n_{\mathrm{n}}$ is neutron density. Clearly cooling occurs much more rapidly than flux expulsion until ages $\gtrsim 10^{6}$ yr when $T<10^{8} \mathrm{~K}$. As a result, magnetic field cannot be expelled from macroscopic regions and is essentially frozen in nuclear matter. A NS core remains in a (metastable) magnetized state (see left side of Fig. 2) even though the minimum energy state is one with a flux-free configuration. We note that, in order to maximize the effectiveness of flux expulsion in comparison to cooling, we use a model which simulates slow NS cooling and ignore effects that would lead to more rapid cooling.

Finally we estimate the growing size of flux-free nucleation regions by setting $\tau_{\mathrm{OhmH}}=t$ in Eq. (1.1) and solving for $l_{\text {mag }}(t)$. Results are shown in Fig. 3. Flux expulsion creates Meissner state regions of size $\sim 10 \mathrm{~m}$ (for $H / 2 H_{\mathrm{c} 2}=10^{-5}$ ). The fact that the cooling lengthscale $l_{\text {cool }} \gg l_{\text {mag }}$ when $t<10^{6}$ yr indicates the superconducting region expands by a much larger distance than the distance over which magnetic field is expelled.

In summary, we confirmed previous estimates that the core retains its magnetic field (see left side of Fig. 2) even though the minimum energy state is one in which magnetic flux is expelled due to the Meissner effect. This is because a dynamical NS cools so rapidly that the superconducting region expands much faster than the field can be expelled by any known processes. To produce a large region in the core devoid of magnetic field, the field must diffuse over macroscopic scales of order a kilometer or more, and the timescale for such field diffusion is $\gtrsim 10^{7} \mathrm{yr}$. At $10^{6} \mathrm{yr}$, the size of flux-free regions is probably $<10 \mathrm{~m}$ and at most $\sim 100 \mathrm{~m}$ (see Fig. 3). This suggests that there is not significant magnetic field evolution in the core of NSs younger than at least $10^{7} \mathrm{yr}$ [see Eq. (1.1); see also Elfritz et al. 2016]. Our results apply to NSs with $H>10^{11} \mathrm{G}$, including magnetars, most of which have $H \gtrsim 10^{14} \mathrm{G}$. Thus for observed magnetars with age $<10^{5} \mathrm{yr}$, there is a limit to the amount of field decay that can occur if the magnetic field in the crust is anchored in the core.

W.C.G.H. appreciates use of computer facilities at KIPAC. W.C.G.H. and N.A. acknowledge support through grant ST/M000931/1 from STFC in the UK. V.G. is supported by a McGill Space Institute postdoctoral fellowship and Trottier Chair in Astrophysics and Cosmology. 


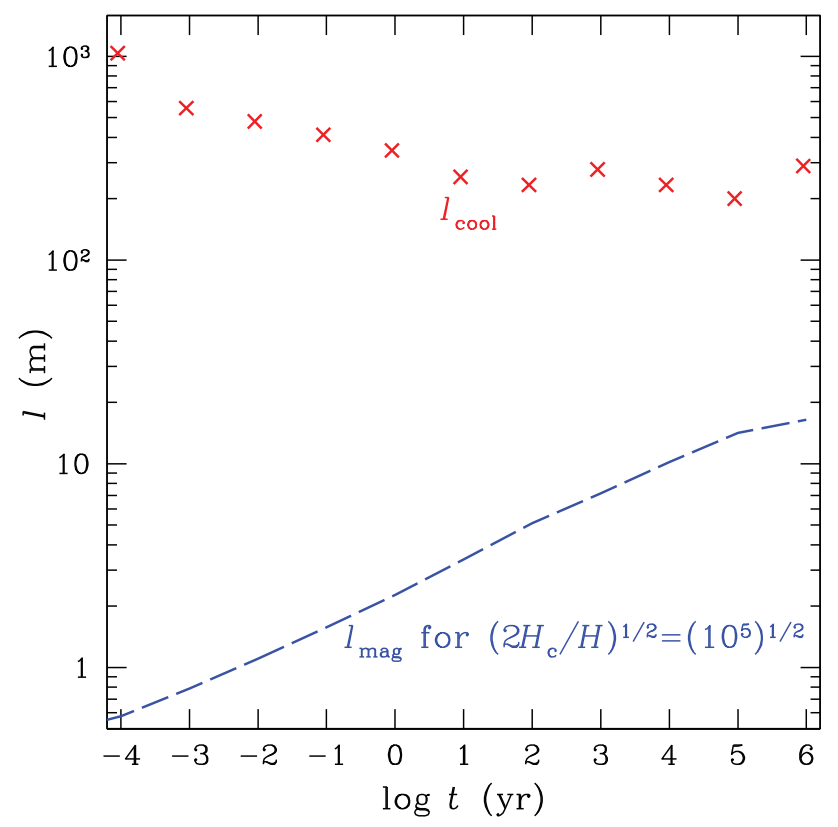

Figure 3. Crosses are radial distance over which the superconducting region grows at each cooling epoch and we define as cooling lengthscale $l_{\text {cool }}$. Dashed line is the lengthscale $l_{\text {mag }}$ obtained using Eq. (1.1) and setting $\tau_{\mathrm{OhmH}}=t . H / 2 H_{\mathrm{c} 2}=10^{-5}$ is assumed.

\section{References}

Akmal, A., Pandharipande, V. R., \& Ravenhall, D. G. 1998, Phys. Rev. C, 58, 1804

Baym, G. 1970, Neutron Stars (Copenhagen: Nordita)

Baym, G., Pethick, C., \& Pines, D. 1969, Nature, 224, 673

Chen, J. M. C., Clark, J. W., Davé, R. D., \& Khodel, V. V. 1993, Nucl. Phys. A, 555, 59

Elfritz, J. G., Pons, J. A., Rea, N., Glampedakis, K., \& Viganò, D. 2016, MNRAS, 456, 4461

Graber, V., Andersson, N., \& Hogg, M. 2017, Int. J. Mod. Phys. D, 26, 1730015

Ho, W. C. G., Glampedakis, K., \& Andersson, N. 2012, MNRAS, 422, 2632; erratum: 2012, MNRAS, 425, 1600

Ho, W. C. G., Andersson, N., \& Graber, V. 2017, Phys. Rev. C, submitted

Page, D., Lattimer, J. M., Prakash, M., \& Steiner, A. W. 2004, ApJS, 155, 623

Page, D., Lattimer, J. M., Prakash, M., \& Steiner, A. W. 2014, in: K.H. Bennemann, J.B. Ketterson (eds.), Novel Superfluids, vol. 2, (Oxford: Oxford University Press), p. 505

Potekhin, A. Y., Pons, J. A., \& Page, D. 2015, Space Sci. Revs, 191, 239

Sauls, J. A. 1989, in: H. Ögelman, E.P.J. van den Heuvel (eds.), Timing Neutron Stars (New York: Kluwer Academic), p. 457

Tinkham, M. 1996, Introduction to Superconductivity, 2nd ed. (New York: McGraw-Hill, Inc.)

Yakovlev, D. G. \& Pethick, C. J. 2004, ARAA, 42, 169 